\title{
Genetic Testing in Patients with Neurodevelopmental Disorders: Experience of 511 Patients at Cincinnati Children's Hospital Medical Center
}

\author{
Xiaoli Du ${ }^{1}$ Jennifer Elaine Glass ${ }^{1} \cdot$ Stephanie Balow ${ }^{1,2} \cdot$ Lisa M. Dyer $^{1,2} \cdot$ Pamela A. Rathbun $^{1,3} \cdot$ Qiaoning Guan $^{1,2}$. \\ Jie Liu ${ }^{1,2} \cdot$ Yaning $\mathrm{Wu}^{1,2} \cdot$ D. Brian Dawson ${ }^{1,2}$. Lauren Walters-Sen ${ }^{1,2} \cdot$ Teresa A. Smolarek ${ }^{1,2} \cdot$ Wenying Zhang $^{1,2}$
}

Accepted: 18 October 2021 / Published online: 13 November 2021

(c) The Author(s) 2021

\begin{abstract}
Our institution developed and continuously improved a Neurodevelopmental Reflex (NDR) algorithm to help physicians with genetic test ordering for neurodevelopmental disorders (NDDs). To assess its performance, we performed a retrospective study of 511 patients tested through NDR from 2018 to 2019. SNP Microarray identified pathogenic/likely pathogenic copy number variations in 27/511 cases (5.28\%). Among the 484 patients tested for Fragile X FMR1 CGG repeats, a diagnosis $(0.20 \%)$ was established for one male mosaic for a full mutation, a premutation, and a one-CGG allele. Within the 101 normocephalic female patients tested for $M E C P 2$, two patients were found to carry pathogenic variants (1.98\%). This retrospective study suggested the NDR algorithm effectively established diagnoses for patients with NDDs with a yield of 5.87\%.
\end{abstract}

Keywords Autism spectrum disorder (ASD) $\cdot$ Copy number variant $(\mathrm{CNV}) \cdot$ Fragile $\mathrm{X} \cdot \mathrm{MECP} 2 \cdot$ Neurodevelopmental disorders

Neurodevelopmental disorders (NDDs) are a group of disorders with impairments of the growth and development of the central nervous system, including autism spectrum disorders (ASD), intellectual disability (ID), developmental delay (DD), etc. NDDs can present as neuropsychiatric problems, impaired motor function, learning difficulties, language delay or non-verbal communication and affect $>3 \%$ of children worldwide (Mithyantha et al., 2017; South et al., 2013; Waggoner et al., 2018). Determining the genetic etiology of a child's NDD can ensure early interventions, access to appropriate services, prognostic information, and accurate recurrent risk assessment. Genetic testing has been recommended by several professional societies, such as

Wenying Zhang

Wenying.Zhang@cchmc.org

1 Division of Human Genetics, Cincinnati Children's Hospital Medical Center, 3333 Burnet Avenue, Cincinnati, OH 45229, USA

2 Department of Pediatrics, University of Cincinnati College of Medicine, Cincinnati, OH, USA

3 Present Address: Pathology and Laboratory Medicine, Ann and Robert H. Lurie Children's Hospital of Chicago, Chicago, IL, USA the American College of Medical Genetics and Genomics (ACMG), the American Academy of Neurology (AAN) and Child Neurology Society (CNS), and the American Academy of Pediatrics (AAP), for patients with NDDs (Filipek et al., 2000; Hyman et al., 2020; Manning et al., 2010; Michelson et al., 2011; Miller et al., 2010; Moeschler et al., 2014; Schaefer et al., 2013; South et al., 2013). However, despite recommendations for genetic testing in children with ASD/ID/DD, a survey study of 3371 families with children suffering from ASD/ID/DD in the United States showed the majority $(68 \%)$ of these children did not undergo recommended genetic testing (Kiely et al., 2016).

Genetic testing for NDDs can be difficult for clinicians to navigate, especially for non-geneticists, and expensive for families and the health care system to afford. Chromosome microarray (CMA) for copy number changes has been recommended as the first-tier clinical diagnostic test for children with NDDs (Manning et al., 2010; Miller et al., 2010; South et al., 2013; Waggoner et al., 2018). Fragile X syndrome is one of the most common causes of X-linked intellectual disability and is caused by an expansion of a trinucleotide repeat region in the promoter of the FMRI gene (Mannermaa et al., 1996; Mila et al., 2018). Rett syndrome is caused by mutations in the $M E C P 2$ gene and is a known 
genetic cause of X-linked autism and ID in normocephalic females (Vidal et al., 2019). Pathogenic variants in PTEN have been reported with a prevalence of $8.3 \%$ and $12.2 \%$ in the ASD-macrocephaly and DD/ID-macrocephaly populations (Varga et al., 2009).

Historically, most patients with NDDs at our institution had concurrent orders for Fragile $\mathrm{X}$ testing, chromosome analysis, and SNP microarray. MECP2 test was only ordered for female children with typical Rett syndrome cognitive and neurologic phenotypes. The patient with atypical Rett syndrome might not be diagnosed by molecular technology (Schaefer et al., 2013). We noticed few orders for PTEN sequencing in patients with NDDs and macrocephaly, likely due to unawareness of the availability of PTEN testing and the complexities of ordering a sendout test.

Therefore, to streamline test ordering and decrease the cost of genetic testing in patients with NDDs, we developed a more complete, user-friendly, and cost-efficient reflexive testing algorithm, named the Neurodevelopmental Reflex (NDR) genetic test. The original NDR algorithm was separated for patients with or without macrocephaly. Those without macrocephaly started with CMA, and proceeded to Fragile $\mathrm{X}$ analysis if CMA was not positive (normal, likely benign, or variant of unknown clinical significance). Those with macrocephaly, as stated by the ordering clinician, started with PTEN sequencing. If PTEN sequencing was normal or found only a variant of unknown clinical significance, testing proceeded to Fragile $\mathrm{X}$ analysis. If Fragile $\mathrm{X}$ testing was negative for a full mutation (normal, grey zone, or premutation) then testing proceeded to CMA. After utilizing this algorithm for a year, we analyzed our outcomes and determined that PTEN sequencing was having a far lower than expected positivity rate in this population, and the algorithm was reformulated to improve its efficiency.

The improved NDR (Fig. 1), which we are evaluating in this study, puts CMA as the first line test for all, with Fragile $\mathrm{X}$ as the 2nd line for all (if CMA is not positive). If Fragile $\mathrm{X}$ analysis was negative for a full mutation (normal, grey zone or premutation repeats), patients noted to be macrocephalic would receive $P T E N$ sequencing and normocephalic female patients would receive $M E C P 2$ sequencing.

In the current analysis, we hope to share our experience in the genetic diagnosis of patients with NDDs while we examine and summarize the clinical utility of this reflexive testing algorithm, which aligns with testing recommended by the ACMG for the genetic diagnosis of patients with NDDs (Moeschler et al., 2014; Schaefer et al., 2013; South et al., 2013). We hope to provide a model of how to streamline a genomic testing algorithm that meets ACMG recommendations in which pediatricians can order a single test for children with NDDs.

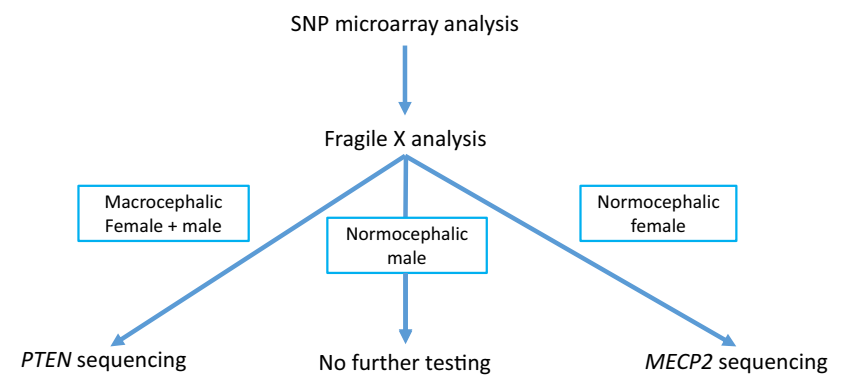

Fig. 1 The algorithm of Neurodevelopmental Reflex (NDR) genetic testing. In this NDR algorithm, the SNP microarray analysis is the first-tier testing, followed by Fragile $\mathrm{X}$ analysis, and the PTEN or $M E C P 2$ gene sequencing. PTEN gene sequencing is applied to macrocephalic patients (as reported by the ordering clinician) with both non-positive SNP microarray and Fragile X testing results. MECP2 gene sequencing is applied only to normocephalic female patients with both non-positive SNP microarray and Fragile X testing results

\section{Methods}

\section{Patient's Enrollment}

A retrospective study of 511 individuals who had a NDR reflex panel ordered through Cincinnati Children's Hospital Medical Center (CCHMC) from January 2018 to April 2019 was conducted to evaluate the performance of the test and positivity rate. This study was approved by CCHMC Institutional Review Board. Subjects' data were obtained in a de-identified state. In this investigation, the patient's ages ranged from 3 months to 35 years old and the sex ratio of male/female was $2.8: 1$. Testing was ordered by clinicians from a broad range of specialties and test indications were extracted from the patients' medical record.

\section{DNA Extraction and SNP Microarray Analysis}

Genomic DNA was extracted either automatically using the Chemagic ${ }^{\mathrm{TM}}$ Magnetic Separation Module I instrument (PerkinElmer, Waltham, MA) or manually using Qiagen Puregene kits (Qiagen, Germany). Microarray analysis was performed using the Infinium Assay with the Illumina CytoSNP-850Kv1.2 BeadChip platform (Illumina, San Diego, CA).

\section{Fragile X}

Isolated genomic DNA was assayed for CGG-repeat expansion of the FMRI locus by analysis of DNA fragments generated by the AmplideX FMR1 polymerase chain reaction (PCR) and separated by capillary electrophoresis ABI 3500XL Genetic Analyzer (Applied Biosystems, Foster city, 
CA). Normal and mutation categories of $F M R 1$ alleles were determined according to the ACMG guidelines with the normal repeat size as $5-44$, gray zone as $45-54$, premutation as 55-200, and full mutation $>200$. Full mutations were confirmed by Southern blot.

\section{PTEN and MECP2 PCR and Sequencing}

The entire coding region and exon/intron boundaries of genes were analyzed by PCR and bidirectional sequencing for PTEN (NM_000314.4) and MECP2 (NM_004992.2) according to manufactory instructions (Roche, Indianapolis, IN).

\section{Blood Chromosomes}

Chromosomal analysis was performed according to standard procedures using GTG-banding.

Detailed methodologies are listed in the supplementary data and available on request.

\section{Results}

The NDR algorithm sets SNP Microarray as the first-tier test and reflexes to Fragile $\mathrm{X}$ testing if the result is not positive, then reflexes to $M E C P 2$ sequencing for normocephalic female patients and PTEN sequencing for patients with macrocephaly (a head circumference $>98 \%$ ile) if the Fragile X result is not full mutation (Fig. 1). Common clinical indications for patients in our cohort included autism spectrum disorders, developmental delay, speech delay, intellectual disability, gross motor delay, mixed receptive-expressive language disorder, epilepsy, and/or dysmorphic features. We analyzed variants detected by this NDR algorithm on patients with neurodevelopmental disorders with samples received from January 2018 to April 2019 at CCHMC. A total of 511 patients (age 3 months -35 years old, median 3 years) were tested. 376 (73.6\%) were male and 135 (26.4\%) were female. More than $36 \%$ patients (188 out of 511) were in the age group of 2 to 3 years. $22.3 \%$ patients (114 out of a

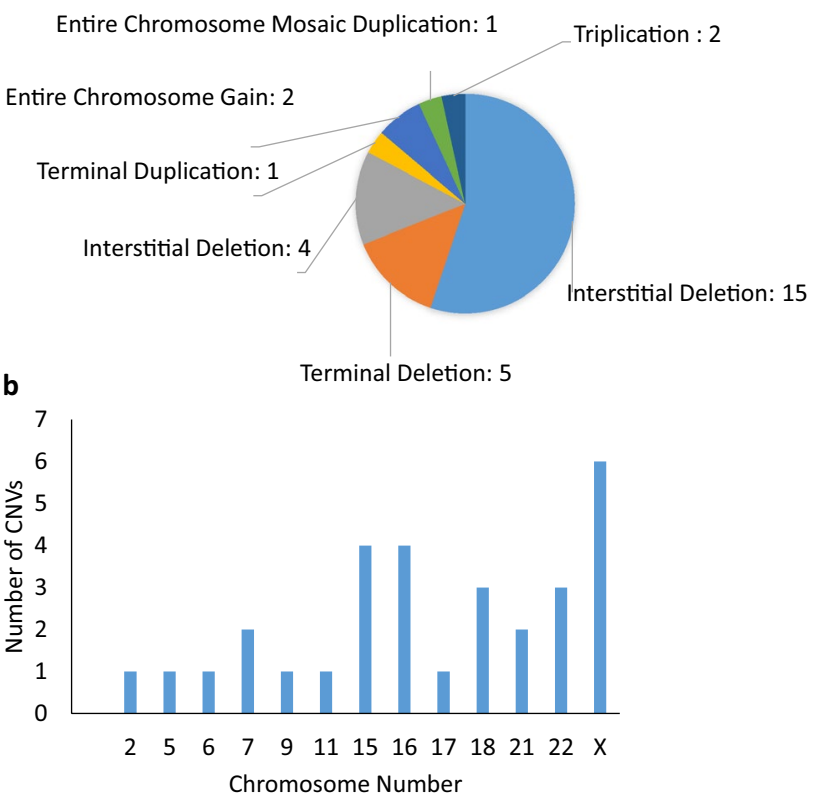

Fig. 3 Clinically significant CNVs identified by SNP microarray analysis in 511 patients. a Classifications and frequency of 30 reportable CNVs in 27 patients through SNP microarray analysis. The majority of CNVs are interstitial deletions (15/30), followed by terminal deletions (5/30), interstitial duplications (4/30), entire chromosome gain and mosaic duplications (3/30). b Accumulated numbers of CNVs on different chromosomes. The most often affected chromosomes are $\mathrm{X}, 15$ or 16

511) were in the age group of 4 to 5 years. $15.8 \%$ patients (81 out of 511) were 6 to 10 years old. $14.8 \%$ patients ( 76 out of 511) were below 2 years (Fig. 2).

$2826 \mathrm{CNV}$ variants were identified through SNP Microarray analysis in the 511 patients. $30 \mathrm{CNVs}$ were pathogenic/likely pathogenic and explained the patient's clinical phenotype (1.06\%). $4.5 \%$ were CNVs of uncertain clinical significance (127). SNP Microarray results provided a diagnosis with clinical significance or likely clinical significance in 27 out of 511 cases (5.28\%), and uncertain clinical significance in 53 out of 511 cases (10.37\%). The majority of the $30 \mathrm{CNVs}$ from 27 patients reported were chromosome
Fig. 2 Description of the 511 patient's cohort from January 2018 to April 2019. a A total of 511 patients tested at our institution from January 2018 to April 2019 included 376 males and 135 females. b Age ranged from a few months to 35 years old. The majority of the patients were under 5 years old
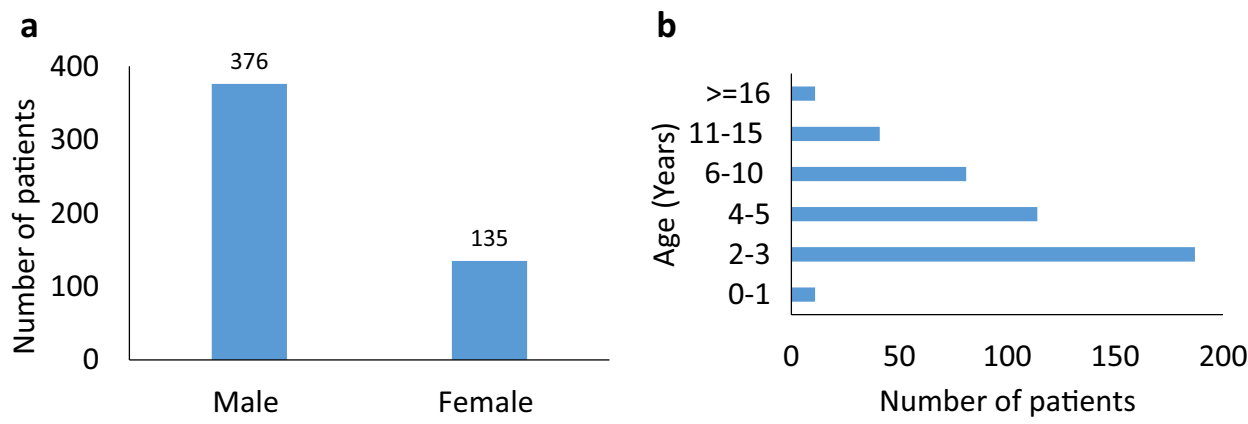
interstitial deletions $(15 / 30)$, followed by terminal deletions (5/30), interstitial duplications (4/30), entire chromosome gain (2/30), and triplication (2/30) (Fig. 3a). The most frequent CNVs identified in this study were from chromosome $\mathrm{X}, 15$ or 16 (Fig. 3b). The most commonly seen syndrome diagnosed by CMA in this study is $15 \mathrm{q} 13.3$ microdeletion syndrome from four patients (Pts 283, 361, 506, 453, Table 1).

There are 30 pathogenic/likely pathogenic CNVs detected in 27 patients with a possible genetic diagnosis. Some patients carry more than one pathogenic/likely pathogenic CNVs. One patient (Pt 319, Table 1) with global developmental delay, speech delay, dysmorphic craniofacial features was identified with a $1.7 \mathrm{Mb}$ duplication of $5 \mathrm{p} 15.33$ and a $2.2 \mathrm{Mb}$ deletion of $18 \mathrm{p} 11.32$, which corresponded to a derivative chromosome 18 due to an unbalanced translocation between the short arms of chromosomes 5 and 18 . Another patient was identified with two terminal deletions $(1.3 \mathrm{Mb}$ and $4.3 \mathrm{Mb})$ on both ends of chromosome 18. This patient presented with club foot, ptosis, speech disturbance, intermittent exotropia, balance problem, weakness, lack of coordination, mixed receptive-expressive language disorder, fine motor impairment, sensory processing difficulty and global developmental delay (Pt 510, Table 1; Fig. 4a). A ring 18 chromosome was suspected and was confirmed by the follow-up chromosome analysis on this patient with a karyotype of 46,XX,r(18)(p11.32q23) (Fig. 4b).

According to the NDR workflow, Fragile $X$ tests were performed in 484 patients (94.72\%). Eight patients showed abnormal FMRI CGG expansion size with repeats numbers including gray zone (45-54) (6 patients), premutation (55-200) (1 patient), and full mutation (>200) (1 patient). This full mutation male patient clinically presented with mixed receptive-expressive language disorder, fine motor development delay and tracheoesophageal fistula. He was identified to have mosaic FMRI mutation alleles including a full mutation allele ( $>200$ CGG repeats), a premutation allele (160 repeats), and a third allele with a smaller than normal repeat size in the 5' UTR of FMRI (Fig. 5a). The full mutation allele identified in this individual exhibited an abnormal methylation pattern of the $F M R I$ gene and this was confirmed by Southern blot analysis (Fig. 5b). Sanger sequencing analysis of the third allele showed that there was a 83-bp deletion containing CGG repeats and an insertion of a single base "A" which resulted in only one CGG repeat in the 5' UTR of the FMRl gene (Fig. 5c). By report, his maternal second cousin had Fragile $\mathrm{X}$ syndrome. There was no report of tremor or ataxia in grandfather, and there was no report of primary ovarian insufficiency in other female family members.

The $M E C P 2$ test has been more utilized after we launched the NDR algorithm. In the past, when the MECP 2 test was just a stand-alone sequencing test, it was ordered, on average, on about 22 patients per year. After the launch of NDR algorithm, it has been ordered 3-4 times more than before as part of the NDR algorithm. A total of 101 patients (19.76\%) were reflexed to $M E C P 2$ sequencing analysis in our cohort. Two patients had heterozygous pathogenic missense variants c.467A > G (p.Asp156Gly) and c.473C > T (p.Thr158Met), respectively, which leads to a diagnosis of Rett syndrome. Two other patients were found to have a heterozygous variant of uncertain significance, c.824 T >C (p.Val275Ala) and c.-187_-186del (Table 2). Another patient detected with an abnormal MECP2 result was a 12 year-old male patient with macrocephaly, epilepsy, and ASD. First-tier CMA analysis detected an $862 \mathrm{~Kb}$ duplication at $\mathrm{Xq} 28$, which included $M E C P 2$, and he was subsequently diagnosed with MECP2 Duplication Syndrome (Pt 198, Table 1). For the 99 patients tested for PTEN sequencing analysis, all of them returned with negative results.

Through reviewing the results of our NDR reflective tests in the 511 patients with NDDs, we found that the diagnostic yield of NDR was $5.87 \%$ in our patient cohort, and the most often causes of NDDs were 15q13.3 microdeletion syndrome, 22q11.2 deletion syndrome, 16p11.2 duplication syndrome, as well as pathogenic variants, including CNVs, in $M E C P 2$.

\section{Discussion}

Neurodevelopmental disorders are the most common medical conditions in pediatric population. Identifying the underlying etiology is important in the care of patients with NDDs including early intervention and clinical management, directing the patients and families to disease-specific supports and resources, informing prognosis, and recurrent risk assessment. Historically, the majority of NDD patients at our institution had concurrent orders placed for Fragile X testing, chromosome analysis, and SNP microarray analysis. The MECP2 and PTEN sequencing tests were underutilized. This NDR algorithm allows clinicians to place a single order that includes all of the testing recommended by ACMG for patients with NDDs, including testing for copy number changes, Fragile $\mathrm{X}$ syndrome, Rett syndrome, and PTEN related macrocephaly/autism syndrome (Schaefer et al., 2013). This algorithm was particularly helpful for the most common ordering providers who are from the Department of Developmental and Behavioral Pediatrics at CCHMC, who are not geneticists. The NDR was designed as a reflexive algorithm with the test with the highest diagnostic yield done first, then reflexing to downstream tests if prior testing is negative/normal. Starting with the highest yield test, the automatic reflexive nature of this algorithm warrants a saving in health care, comparing to the cost when these tests were ordered concurrently. Ordering these tests 


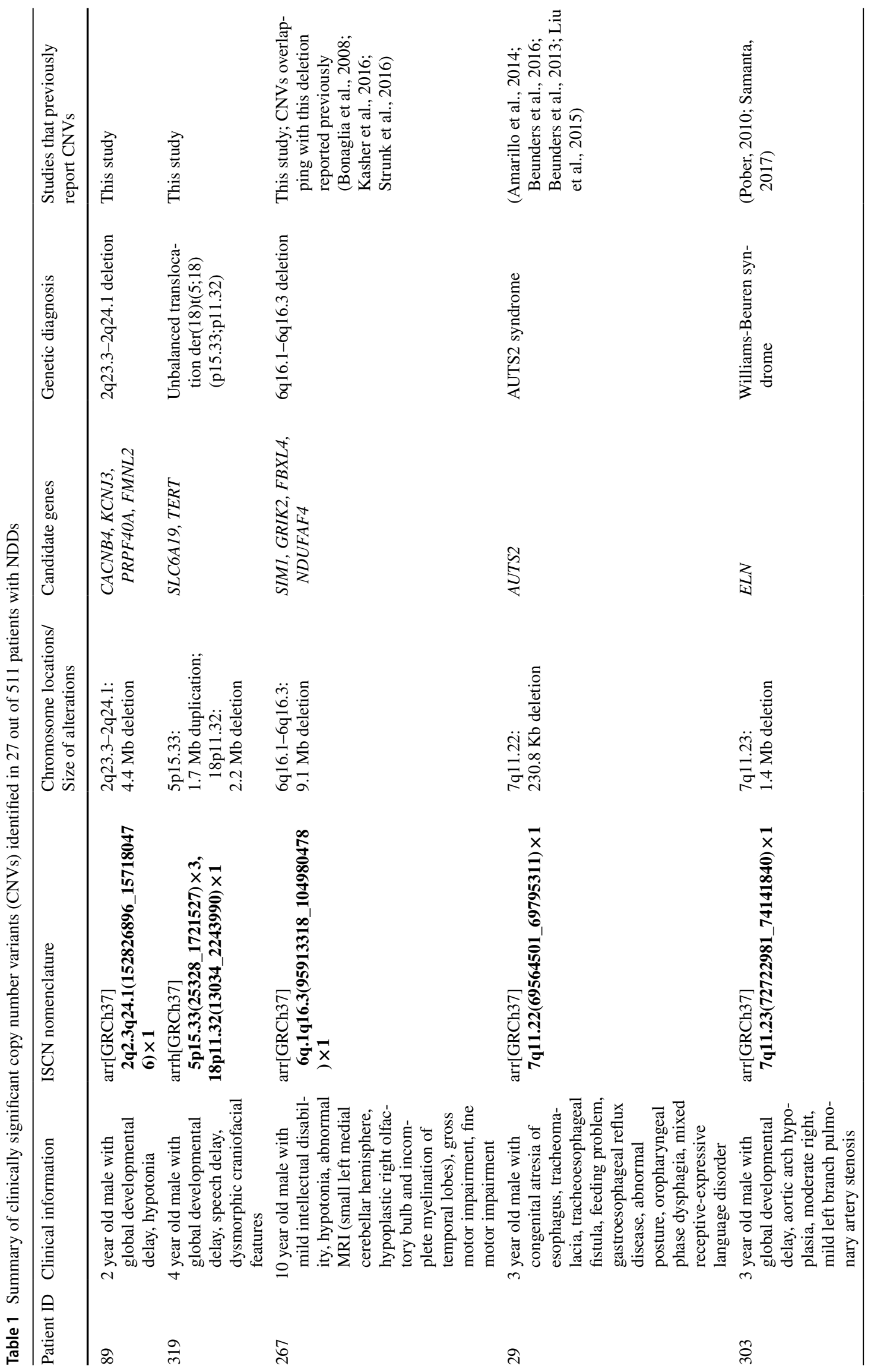




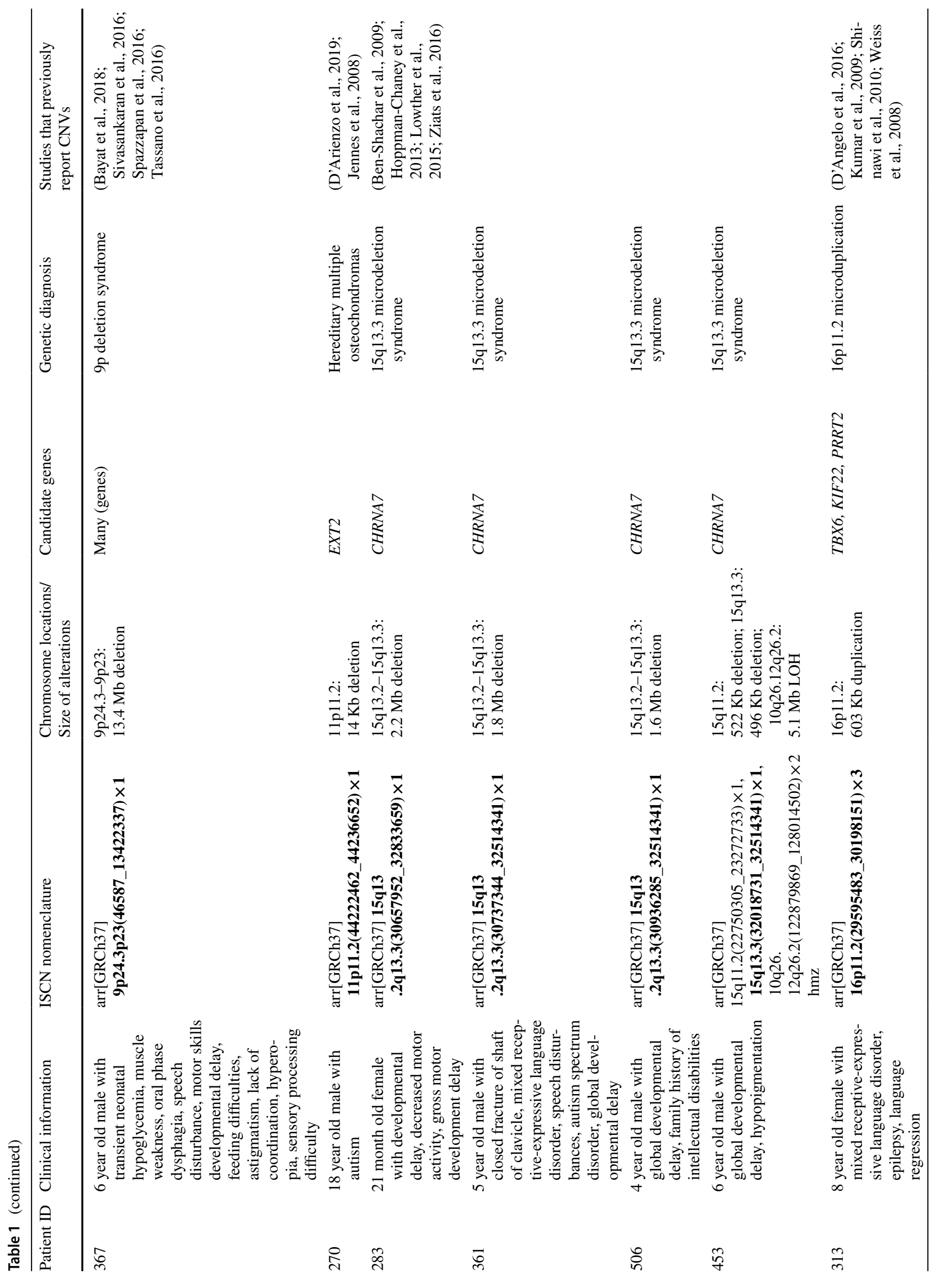




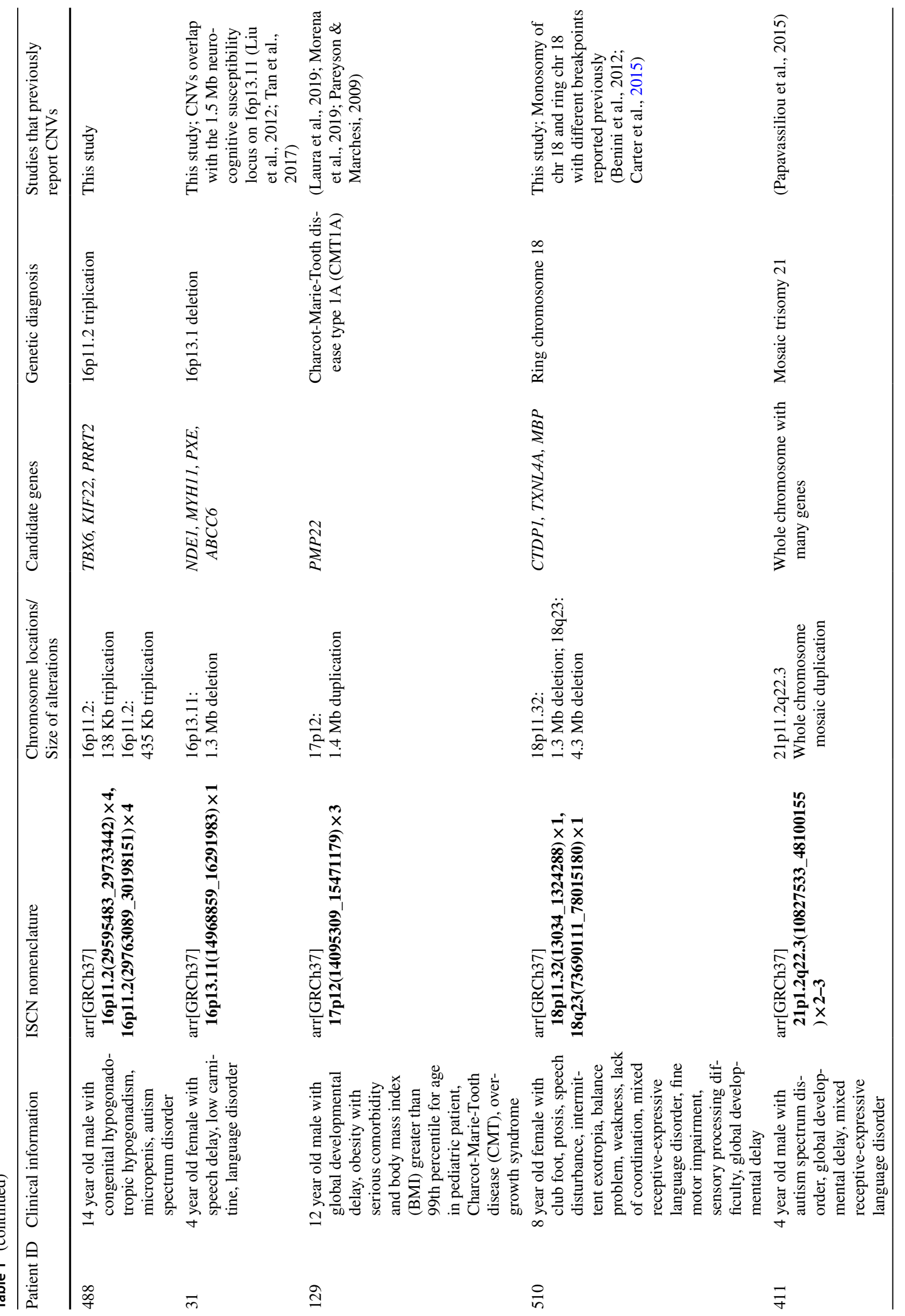




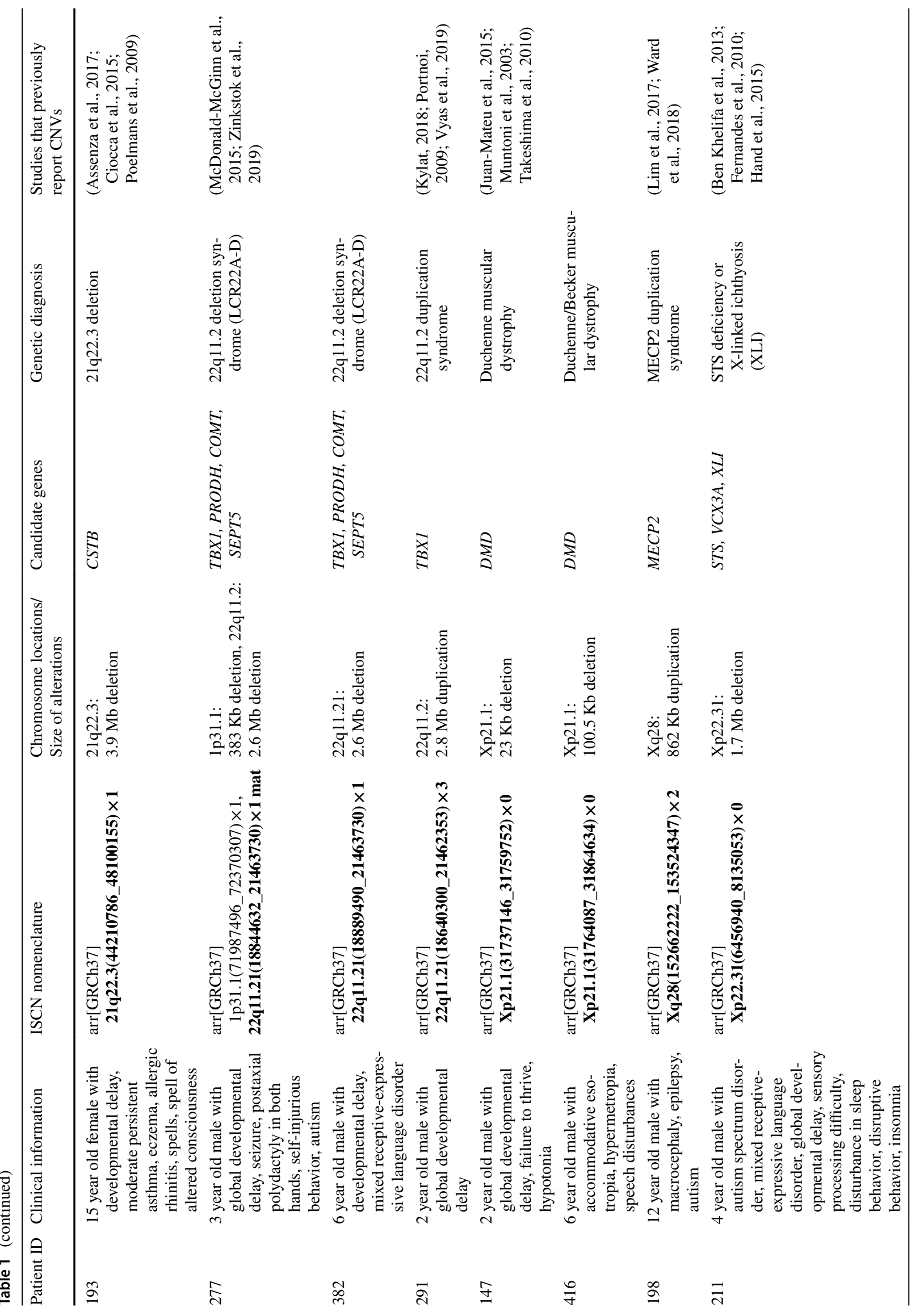




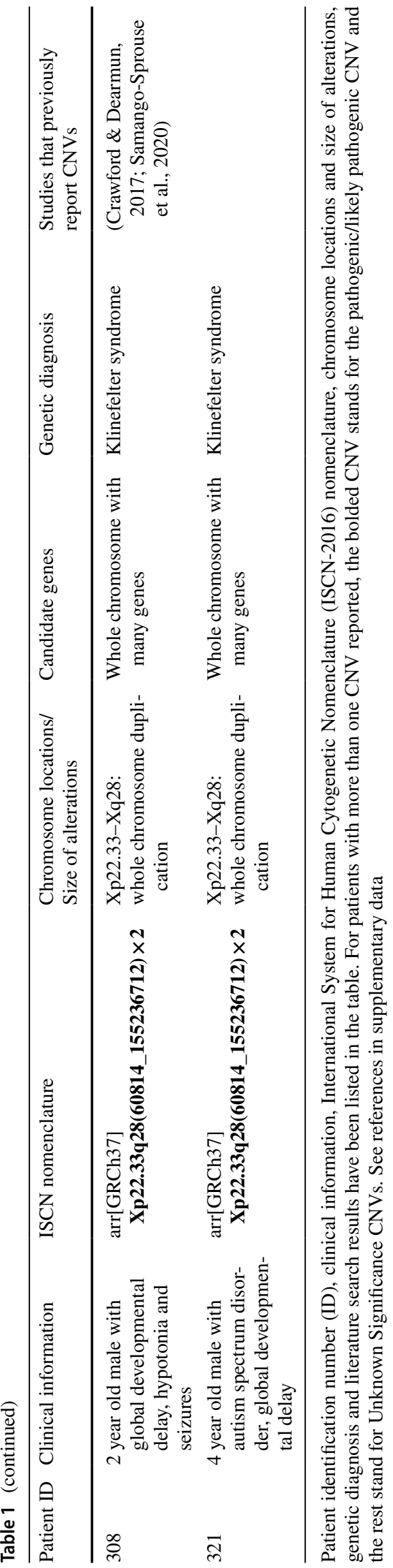

in a sequential order manually will have the same cost of saving effect. However, this automatic reflexive algorithm will save time in the overall diagnostic odyssey and is more user-friendly when compared with the manual sequential order of these tests. The NDR algorithm also promoted a greater utilization of the MECP2 testing in NDD patients, especially for patients with atypical Rett syndrome, and further identified the genetic etiology for two patients in our cohort. These patients might be undiagnosed if the historical routine tests were ordered. Although the PTEN analysis in our cohort did not identify any positive cases, a negative result reduces the likelihood of PTEN as the genetic etiology for a patient with NDD, which is very meaningful for the patient care, since PTEN hamartoma tumor syndrome is associated with increased risk for certain cancers (breast, thyroid, renal cell, etc.).

One of the goals for this study was to review and evaluate the diagnostic yield of clinically ordered NDR tests. Our data show a $5.87 \%$ diagnostic yield, with the majority of positive cases (5.28\%) diagnosed by CMA in the first-tier of testing. It has been reported that the diagnostic yield of CMA ranged from 4.5 to $28.0 \%$ in 19 studies of ID/DD and ranged from $1.5 \%$ to $20.5 \%$ in 11 studies with ASD (Savatt \& Myers, 2021). Although our study did not separate ID/ DD from ASD, the diagnostic yield of CMA in our cohort fell right into the previously reported diagnostic yield range of both. The most often causes of NDDs in our cohort from the first-tier CMA analysis were 15q13.3 microdeletion syndrome, 22q11.2 deletion syndrome, and 16p11.2 duplication syndrome. These findings were consistent with the "hot spots" autism loci reported in the 2013 ACMG practice guideline (Schaefer et al., 2013).

An interesting finding in our cohort that we would like to discuss is the ring chromosome. We detected by CMA and confirmed by high resolution chromosomal analysis one patient carrying a ring chromosome 18 with a karyotype 46,XX,r(18)(p11.32q23). Ring chromosome, which arises following breakage and rejoining in both chromosome arms, has been reported for all human chromosomes with an estimated frequency between 1/30,000 and 1/60,000 (Heydari et al., 2014). Carriers of ring chromosomes may have variable degrees of symptoms, from asymptomatic to serious defects in physical and intellectual development. Common features of patients with ring chromosome syndrome include short stature and developmental delay (Guilherme et al., 2013; Pristyazhnyuk \& Menzorov, 2018). Although ring 18 chromosome with the exact breakpoints has not been reported so far, patients with partial monosomy 18p or ring 18 with different breakpoints have been reported with clinical features including difficulties in resisting infections, holoprosencephaly, micrognathia, tooth decay, ptosis, delayed development, intellectual disability, hypotonia, failure to thrive, short stature with growth hormone deficiencies, 
a Region 1
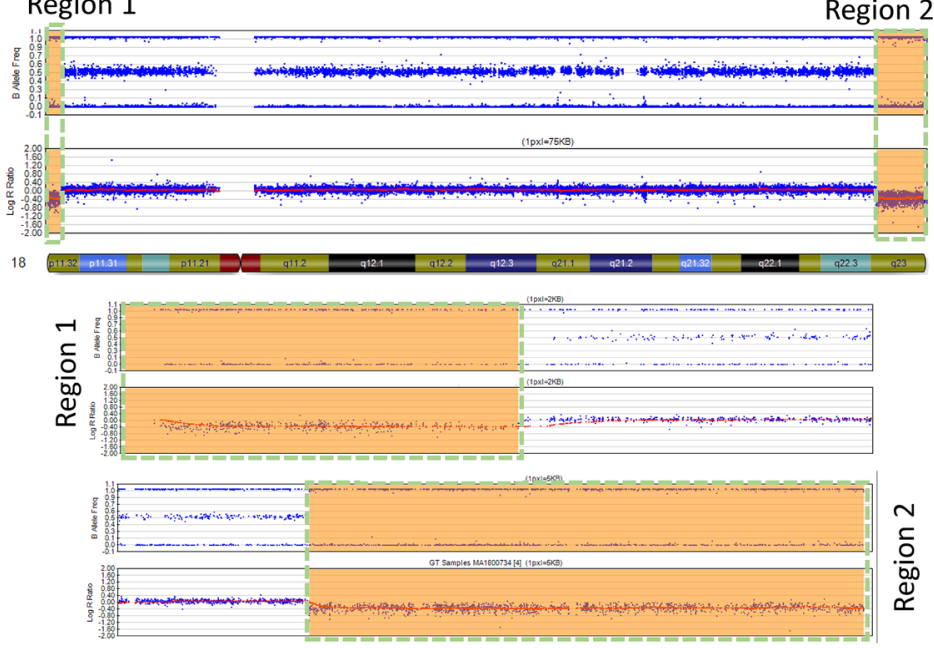

arr[GRCh37] 18p11.32(13034_1324288)×1,18q23(73690111_78015180)x1 b

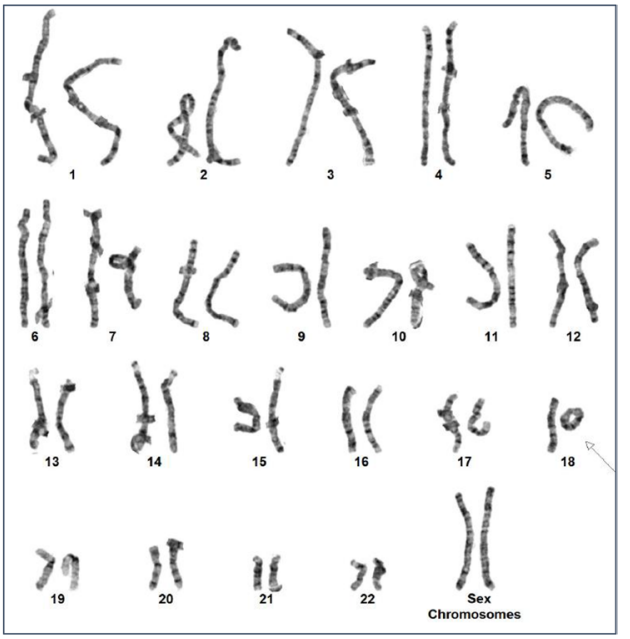

$46, X X, r(18)(p 11.32 q 23)$
Fig. 4 SNP microarray and karyotype analyses identified a ring chromosome 18 in a patient with club foot, ptosis, speech disturbance and global developmental delay. a Results from GenomeStudio shows two terminal deletions for chromosome 18 , which suggested a ring chromo- some 18. Region 1 corresponds to 18p11.32(13034_1324288) $\times 1$ and region 2 corresponds to 18q23(73690111_78015180) $\times 1$. b High resolution blood chromosome confirmed the ring chromosome 18 with a karyotype of $46, \mathrm{XX}, \mathrm{r}(18)(\mathrm{p} 11.32 \mathrm{q} 23)$, which is indicated by the arrow

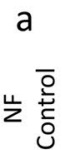

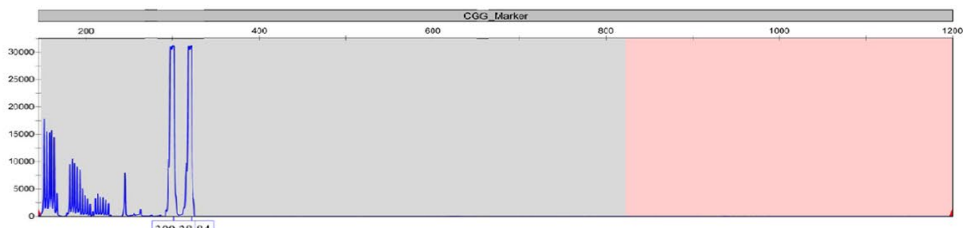

300884
3028
31240
306

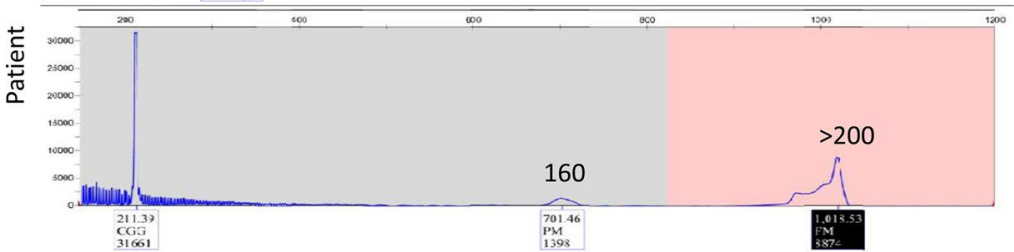

FMR1 deletion of 83bp and insertion of $1 \mathrm{bp}:$ "A"
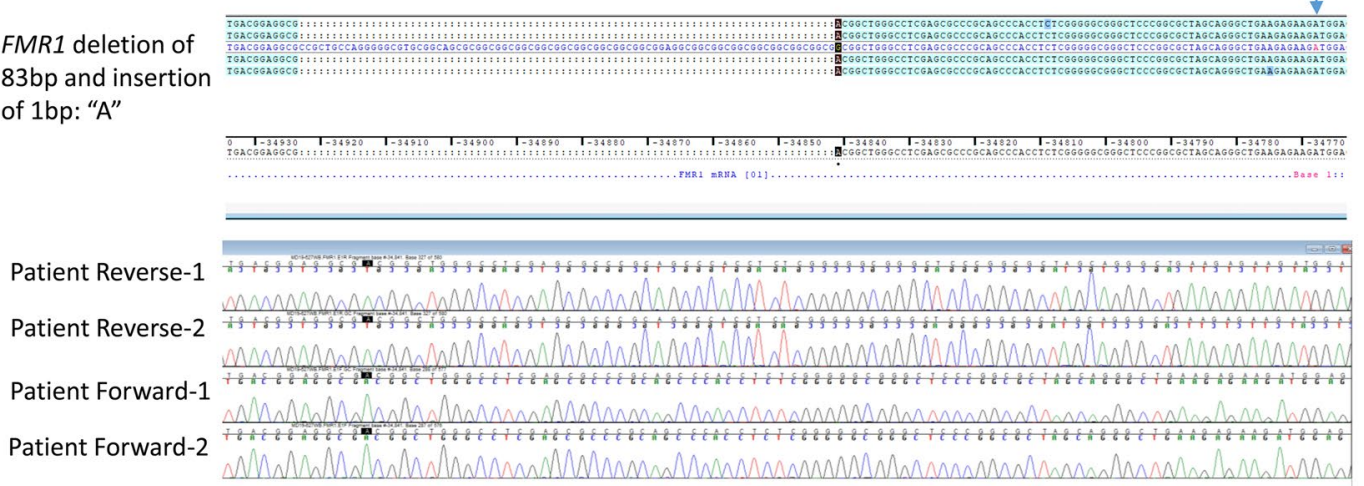

\section{$\sqrt{60 \mathrm{x}}$}

$b$

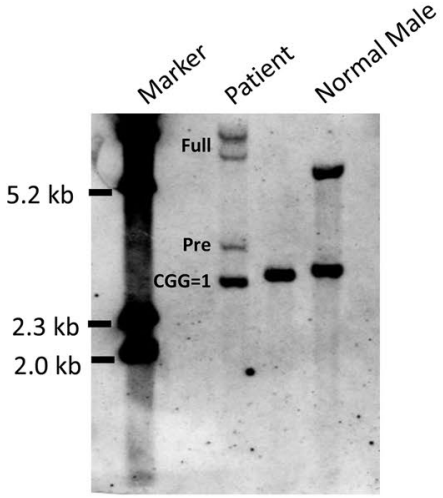

c.1

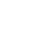




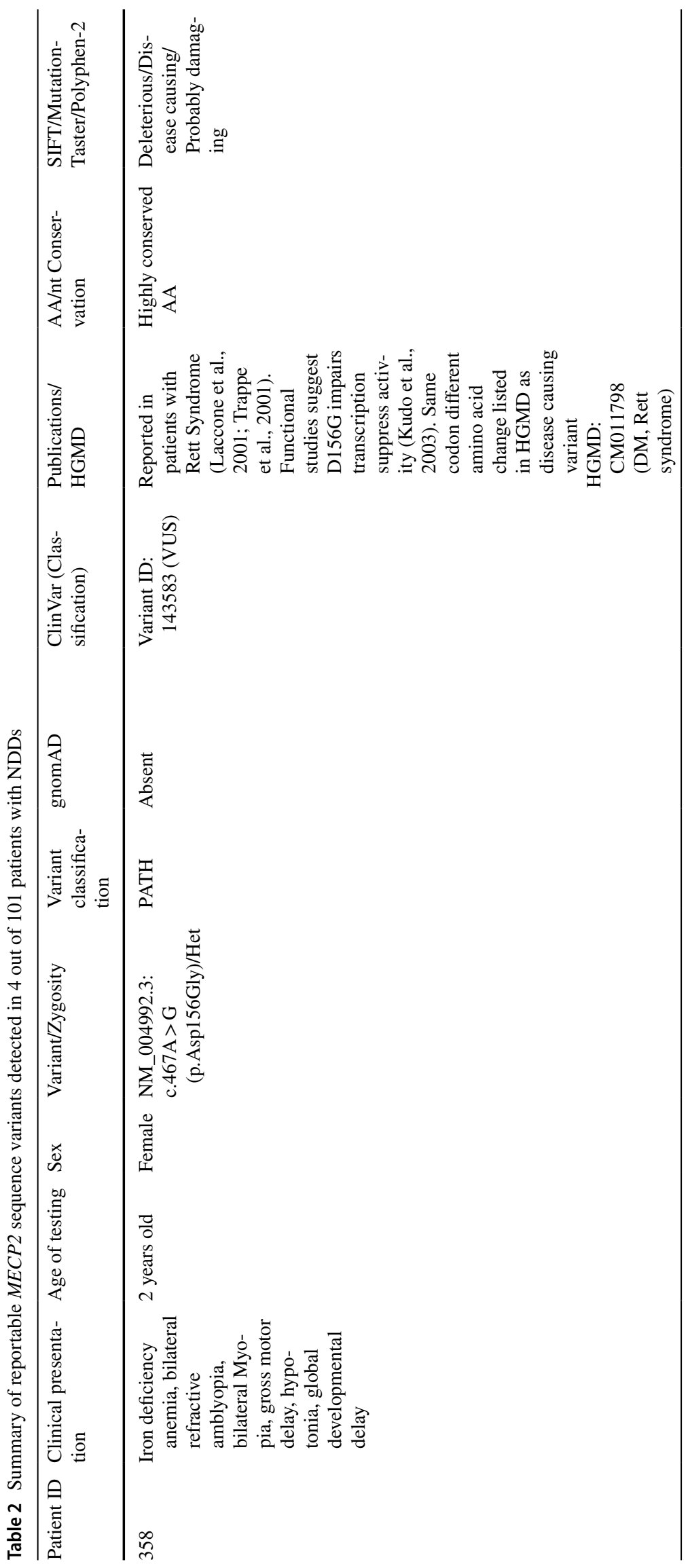




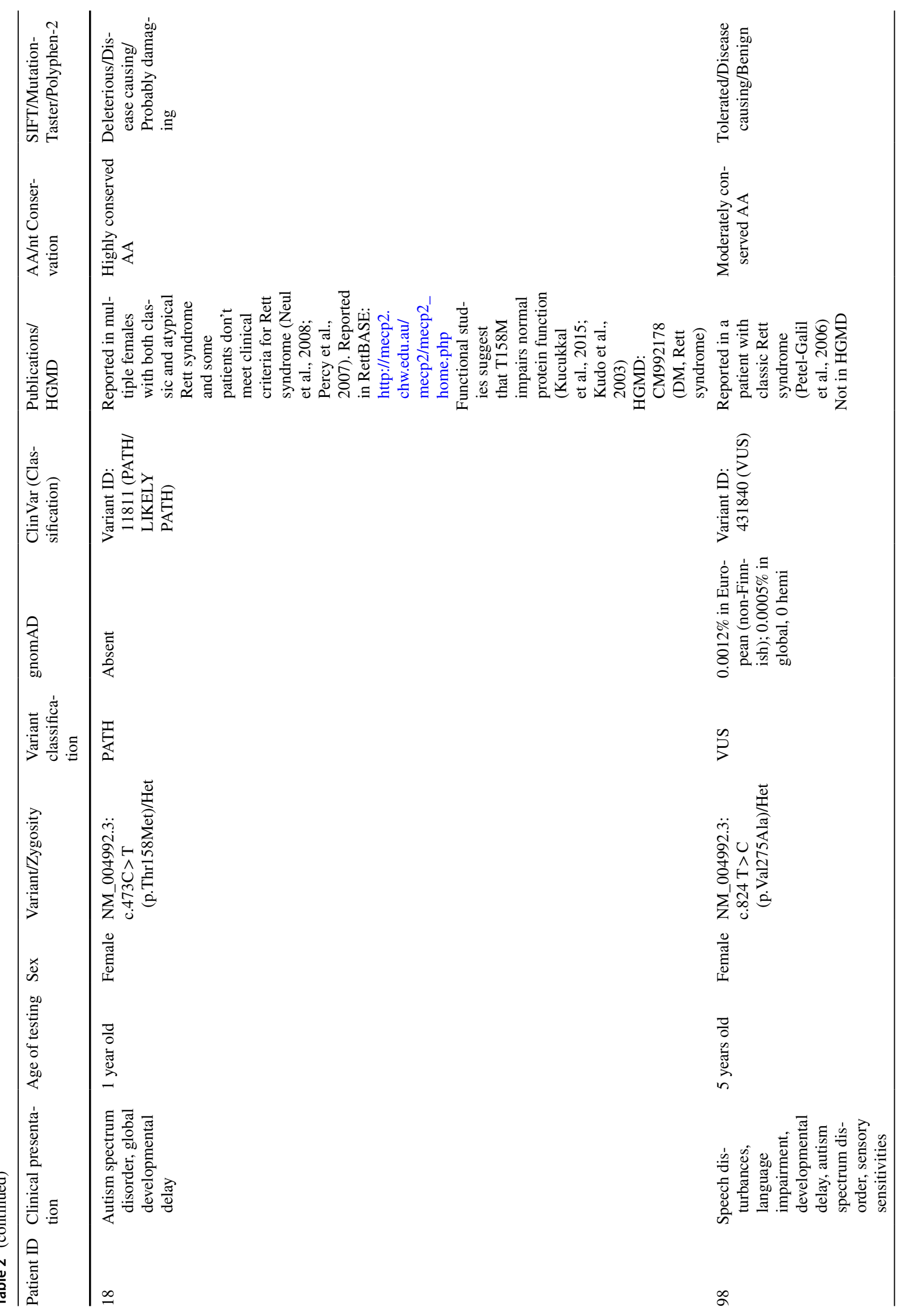




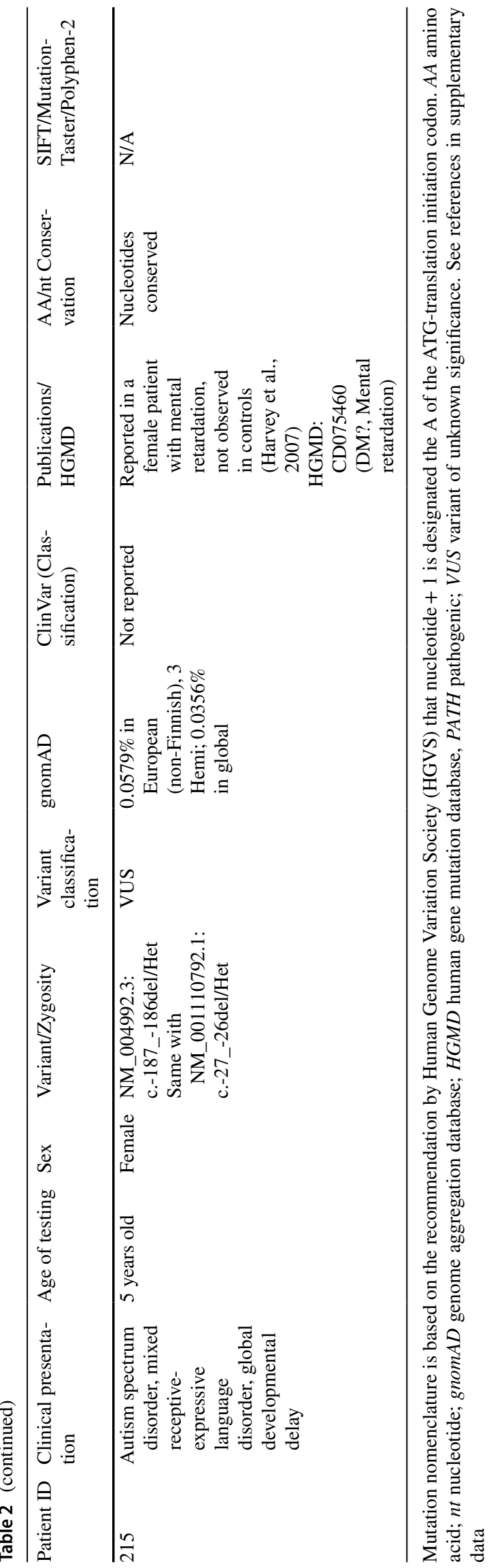

microcephaly, speech problems, hypertelorism, low-set ears, epicanthal folds, and cleft palate (Carter et al., 2015; Chen et al., 2010; Heydari et al., 2014; Stankiewicz et al., 2001; Timur et al., 2004). The signs and symptoms associated with a ring chromosome 18 depend on how much genetic material is lost from each arm of the chromosome. A critical gene is myelin basic protein (MBP; OMIM: 159430), which is located at the deleted region of 18q23 (Harauz et al., 2009). $M B P$ gene encodes a protein which is incorporated in oligodendrocytes and Schwann cells myelin sheets. It has been reported as a candidate gene in a 2.5 year-old male, with an abnormal chromosome karyotype of 46,XY,r(18) (p11.32q21.32), who had overlapping features with our patient, including cleft lip, club foot and mild developmental delay (Heydari et al., 2014).

It was suggested that molecular single gene testing for Fragile X, Rett Syndrome, and PTEN will complement CMA and/or traditional cytogenetics in a clinical setting for neurodevelopmental/ASD disorder diagnosis (Schaefer et al., 2013). In this study, fragile $X$ syndrome (FXS) testing identified mosaic FMRI full mutation alleles in a male patient. This patient carried a full mutation allele, a premutation allele, and an allele with one CGG repeat that resulted from an indel in the $5^{\prime}$ UTR region of the FMR gene. Deletions found in the mosaic state in full mutation males have been reported but are very rare and are typically larger than the deletion identified in this patient (Coffee et al., 2008; de Graaff et al., 1995; de Vries et al., 1993; Goncalves et al., 2016; Mannermaa et al., 1996). The phenotypic consequence of this indel in the mosaic state is currently unknown. Although we only found one patient with FXS, since ASD is present in 50-70\% of individuals with FXS, a negative test result can exclude the diagnosis of FXS and allow subsequent reflex tests or other genetic tests to be performed.

PTEN is a well-known gene associated with ASD and macrocephaly (Butler et al., 2005; Varga et al., 2009; Zhou \& Parada, 2012) and has been recommended by the ACMG practice guideline in identification of the etiology of ASD (Schaefer et al., 2013). Based on the ACMG practice guideline, we included PTEN sequencing in our NDR algorithm. When we initially established the NDR reflexing algorithm, PTEN analysis was set as the first-tier testing for patients who presented with macrocephaly, followed by Fragile $\mathrm{X}$ testing, and finally SNP microarray analysis. However, all the 40 patients with macrocephaly who received $P T E N$ testing returned with negative results (data not shown). Therefore, we adjusted the algorithm as indicated in Fig. 1 to move PTEN sequencing to the third tier. Interestingly, in the current study, none of the 99 patients with macrocephaly were positive for pathogenic variants by PTEN sequence analysis. This "lower than expected" yield from $P T E N$ sequencing may be due to the bias in defining macrocephaly or the fact that many macrocephalic patients 
in our cohort may have NDD other than ASD, which is the main symptom associated with PTEN. While this supports the decision to move PTEN to the end of the algorithm, given the extremely low yield of PTEN testing, we are considering replacing it with a more comprehensive next generation sequencing test which covers more genes and has a higher yield in NDDs. Single gene PTEN sequencing will still be available for clinicians to order if there is a strong clinical indication for a disorder associated with variants in this gene.

In summary, we show here that the NDR algorithm can effectively establish the genetic diagnosis for patients with NDDs, especially using CMA as a first-tier test following the ACMG guideline published in 2010 (Miller et al., 2010). With the advance of next-generation sequencing (NGS) technology and its implementation in clinical genetic laboratories, tests utilizing a combination of NGS and microarray have shown higher diagnostic yield in NDDs. In a recent study, a cohort of 8565 patients with epilepsy and NDDs tested by NGS and aCGH identified a genetic etiology in $15.4 \%$ of patients (Lindy et al., 2018). A meta-analysis comparing the yield of exome sequencing (ES) in NDDs with that of CMA showed that ES's yield was markedly greater than CMA and a consensus was proposed to place ES as the first-tier clinical test in a diagnostic algorithm for unexplained NDDs (Srivastava et al., 2019). Recently, a new ACMG guideline was published recommending that exome and genome sequencing should be considered as a first- or second-tier test for patients with one or more congenital anomalies (CA)/DD/ID (Manickam et al., 2021).

Our laboratory recently launched an ASD/ID/DD exome slice panel with the option to reflex to WES, including trio analysis (proband and parents). We will monitor the performance of this exome slice panel with option reflex to WES test and plan to incorporate it into our NDR algorithm to continuously improve the diagnosis of NDDs. The field of clinical genetic testing is rapidly changing with advances in technology, such as genome sequencing, transcriptome sequencing, genome-wide methylation analysis, etc., which makes additional genetic tests available and affordable and more genes discovered in association with ASD/ID/DD. It is necessary for clinical genetic laboratories to continue to update genetic testing algorithms to increase the diagnosis of NDDs accordingly when new technologies become clinically available.

Supplementary Information The online version contains supplementary material available at https://doi.org/10.1007/s10803-021-05337-6.

Acknowledgments The authors thank the ordering providers that referred patients to our laboratory for NDR tests. The authors also thank the Genetics and Genomics Diagnostic Laboratory staff that performed the NDR tests.
Author Contributions XD, TAS, and WZ conceptualized and designed the study; SB, LMD, PAR, QG, JL, YW, DBD, LW, TAS, and WZ reviewed, interpreted, and reported the NDR test results; XD, JEG, and WZ analyzed data; XD, JEG, and WZ drafted the manuscript; and all authors reviewed and contributed to the manuscript.

Open Access This article is licensed under a Creative Commons Attribution 4.0 International License, which permits use, sharing, adaptation, distribution and reproduction in any medium or format, as long as you give appropriate credit to the original author(s) and the source, provide a link to the Creative Commons licence, and indicate if changes were made. The images or other third party material in this article are included in the article's Creative Commons licence, unless indicated otherwise in a credit line to the material. If material is not included in the article's Creative Commons licence and your intended use is not permitted by statutory regulation or exceeds the permitted use, you will need to obtain permission directly from the copyright holder. To view a copy of this licence, visit http://creativecommons.org/licenses/by/4.0/.

\section{References}

Butler, M. G., Dasouki, M. J., Zhou, X. P., Talebizadeh, Z., Brown, M., Takahashi, T. N.,... Eng, C. (2005). Subset of individuals with autism spectrum disorders and extreme macrocephaly associated with germline PTEN tumour suppressor gene mutations. Journal of Medical Genetics, 42(4), 318-321https://doi.org/10.1136/jmg. 2004.024646

Carter, E., Heard, P., Hasi, M., Soileau, B., Sebold, C., Hale, D. E., \& Cody, J. D. (2015). Ring 18 molecular assessment and clinical consequences. American Journal of Medical Genetics Part A, 167A(1), 54-63. https://doi.org/10.1002/ajmg.a.36822

Chen, C. P., Kuo, Y. T., Lin, S. P., Su, Y. N., Chen, Y. J., Hsueh, R. Y.,... Wang, W. (2010). Mosaic ring chromosome 18, ring chromosome 18 duplication/deletion and disomy 18: Perinatal findings and molecular cytogenetic characterization by fluorescence in situ hybridization and array comparative genomic hybridization. Taiwanese Journal of Obstetrics and Gynecology, 49(3), 327-332https://doi.org/10.1016/S1028-4559(10)60069-1

Coffee, B., Ikeda, M., Budimirovic, D. B., Hjelm, L. N., Kaufmann, W. E., \& Warren, S. T. (2008). Mosaic FMR1 deletion causes fragile $\mathrm{X}$ syndrome and can lead to molecular misdiagnosis: A case report and review of the literature. American Journal of Medical Genetics Part A, 146A(10), 1358-1367. https://doi.org/10.1002/ ajmg.a.32261

de Graaff, E., Rouillard, P., Willems, P. J., Smits, A. P., Rousseau, F., \& Oostra, B. A. (1995). Hotspot for deletions in the CGG repeat region of FMR1 in fragile X patients. Human Molecular Genetics, 4(1), 45-49. https://doi.org/10.1093/hmg/4.1.45

de Vries, B. B., Fryns, J. P., Butler, M. G., Canziani, F., Wesby-van Swaay, E., van Hemel, J. O., ... Niermeijer, M. F. (1993). Clinical and molecular studies in fragile $\mathrm{X}$ patients with a Prader-Willi-like phenotype. Journal of Medical Genetics, 30(9), 761-766https:// doi.org/10.1136/jmg.30.9.761

Filipek, P. A., Accardo, P. J., Ashwal, S., Baranek, G. T., Cook, E. H., Jr., Dawson, G., ... Volkmar, F. R. (2000). Practice parameter: screening and diagnosis of autism: Report of the quality standards subcommittee of the American academy of neurology and the child neurology society. Neurology, 55(4), 468-479https://doi.org/ 10.1212/wnl.55.4.468

Goncalves, T. F., dos Santos, J. M., Goncalves, A. P., Tassone, F., Mendoza-Morales, G., Ribeiro, M. G., ... Santos-Reboucas, C. B. 
(2016). Finding FMR1 mosaicism in Fragile X syndrome. Expert Review of Molecular Diagnostics, 16(4), 501-507https://doi.org/ 10.1586/14737159.2016.1135739

Guilherme, R., Klein, E., Hamid, A., Bhatt, S., Volleth, M., Polityko, A., ... Liehr, T. (2013). Human ring chromosomes - New insights for their clinical significance. Balkan Journal of Medical Genetics, 16(1), 13-20https://doi.org/10.2478/bjmg-2013-0013

Harauz, G., Ladizhansky, V., \& Boggs, J. M. (2009). Structural polymorphism and multifunctionality of myelin basic protein. Biochemistry, 48(34), 8094-8104. https://doi.org/10.1021/bi901005f

Heydari, S., Hassanzadeh, F., \& Hassanzadeh Nazarabadi, M. (2014). Ring chromosome 18: A case report. International Journal of Molecular and Cellular Medicine, 3(4), 287-289. https://www. ncbi.nlm.nih.gov/pubmed/25635256

Hyman, S. L., Levy, S. E., Myers, S. M., Council On Children With Disabilities, S. O. D., \& Behavioral, P. (2020). Identification, evaluation, and management of children with autism spectrum disorder. Pediatrics, 145(1). https://doi.org/10.1542/peds.2019-3447

Kiely, B., Vettam, S., \& Adesman, A. (2016). Utilization of genetic testing among children with developmental disabilities in the United States. The Application of Clinical Genetics, 9, 93-100. https://doi.org/10.2147/TACG.S103975

Lindy, A. S., Stosser, M. B., Butler, E., Downtain-Pickersgill, C., Shanmugham, A., Retterer, K., ... McKnight, D. A. (2018). Diagnostic outcomes for genetic testing of 70 genes in 8565 patients with epilepsy and neurodevelopmental disorders. Epilepsia, 59(5), 1062-1071https://doi.org/10.1111/epi.14074

Manickam, K., McClain, M. R., Demmer, L. A., Biswas, S., Kearney, H. M., Malinowski, J., ... Directors, A. B. O. (2021). Exome and genome sequencing for pediatric patients with congenital anomalies or intellectual disability: An evidence-based clinical guideline of the American College of Medical Genetics and Genomics (ACMG). Genetics in Medicinehttps://doi.org/10.1038/ s41436-021-01242-6

Mannermaa, A., Pulkkinen, L., Kajanoja, E., Ryynanen, M., \& Saarikoski, S. (1996). Deletion in the FMR1 gene in a Fragile-X male. American Journal of Medical Genetics, 64(2), 293-295. https:// doi.org/10.1002/(SICI)1096-8628(19960809)64:2\%3c293::AIDAJMG12\%3e3.0.CO;2-A

Manning, M., Hudgins, L., Professional, P., \& Guidelines, C. (2010). Array-based technology and recommendations for utilization in medical genetics practice for detection of chromosomal abnormalities. Genetics in Medicine, 12(11), 742-745. https://doi.org/ 10.1097/GIM.0b013e3181f8baad

Michelson, D. J., Shevell, M. I., Sherr, E. H., Moeschler, J. B., Gropman, A. L., \& Ashwal, S. (2011). Evidence report: Genetic and metabolic testing on children with global developmental delay: Report of the quality standards subcommittee of the American academy of neurology and the practice committee of the child neurology society. Neurology, 77(17), 1629-1635. https://doi.org/ 10.1212/WNL.0b013e3182345896

Mila, M., Alvarez-Mora, M. I., Madrigal, I., \& Rodriguez-Revenga, L. (2018). Fragile X syndrome: An overview and update of the FMR1 gene. Clinical Genetics, 93(2), 197-205. https://doi.org/ 10.1111/cge. 13075

Miller, D. T., Adam, M. P., Aradhya, S., Biesecker, L. G., Brothman, A. R., Carter, N. P., ... Ledbetter, D. H. (2010). Consensus statement: Chromosomal microarray is a first-tier clinical diagnostic test for individuals with developmental disabilities or congenital anomalies. The American Journal of Human Genetics, 86(5), 749764https://doi.org/10.1016/j.ajhg.2010.04.006

Mithyantha, R., Kneen, R., McCann, E., \& Gladstone, M. (2017). Current evidence-based recommendations on investigating children with global developmental delay. Archives of Disease in
Childhood, 102(11), 1071-1076. https://doi.org/10.1136/archd ischild-2016-311271

Moeschler, J. B., Shevell, M., \& Committee on, G. (2014). Comprehensive evaluation of the child with intellectual disability or global developmental delays. Pediatrics, 134(3), e903-918. https://doi. org/10.1542/peds.2014-1839

Pristyazhnyuk, I. E., \& Menzorov, A. G. (2018). Ring chromosomes: From formation to clinical potential. Protoplasma, 255(2), 439449. https://doi.org/10.1007/s00709-017-1165-1

Savatt, J. M., \& Myers, S. M. (2021). Genetic testing in neurodevelopmental disorders. Frontiers in Pediatrics, 9, 526779. https://doi. org/10.3389/fped.2021.526779

Schaefer, G. B., Mendelsohn, N. J., Professional, P., \& Guidelines, C. (2013). Clinical genetics evaluation in identifying the etiology of autism spectrum disorders: 2013 guideline revisions. Genetics in Medicine, 15(5), 399-407. https://doi.org/10.1038/gim.2013.32

South, S. T., Lee, C., Lamb, A. N., Higgins, A. W., Kearney, H. M., Working Group for the American College of Medical, G., \& Genomics Laboratory Quality Assurance, C. (2013). ACMG standards and guidelines for constitutional cytogenomic microarray analysis, including postnatal and prenatal applications: Revision 2013. Genetics in Medicine, 15(11), 901-909 https://doi.org/ 10.1038/gim.2013.129

Srivastava, S., Love-Nichols, J. A., Dies, K. A., Ledbetter, D. H., Martin, C. L., Chung, W. K., ... Group, N. D. D. E. S. R. W. (2019). Meta-analysis and multidisciplinary consensus statement: Exome sequencing is a first-tier clinical diagnostic test for individuals with neurodevelopmental disorders. Genetics in Medicine, 21(11), 2413-2421https://doi.org/10.1038/s41436-019-0554-6

Stankiewicz, P., Brozek, I., Helias-Rodzewicz, Z., Wierzba, J., Pilch, J., Bocian, E., ... Limon, J. (2001). Clinical and molecular-cytogenetic studies in seven patients with ring chromosome 18. The American Journal of Medical Genetics, 101(3), 226-239https:// doi.org/10.1002/1096-8628(20010701)101:3<226::aid-ajmg1 349>3.0.co;2-\#

Timur, A. A., Sadgephour, A., Graf, M., Schwartz, S., Libby, E. D., Driscoll, D. J., \& Wang, Q. (2004). Identification and molecular characterization of a de novo supernumerary ring chromosome 18 in a patient with Klippel-Trenaunay syndrome. Annals of Human Genetics, 68(Pt 4), 353-361. https://doi.org/10.1046/j.1529-8817. 2004.00095.x

Varga, E. A., Pastore, M., Prior, T., Herman, G. E., \& McBride, K. L. (2009). The prevalence of PTEN mutations in a clinical pediatric cohort with autism spectrum disorders, developmental delay, and macrocephaly. Genetics in Medicine, 11(2), 111-117. https://doi. org/10.1097/GIM.0b013e31818fd762

Vidal, S., Xiol, C., Pascual-Alonso, A., OCallaghan, M., Pineda, M., \& Armstrong, J. (2019). Genetic landscape of rett syndrome spectrum: Improvements and challenges. International Journal of Molecular Science, 20(16). https://doi.org/10.3390/ijms20163925

Waggoner, D., Wain, K. E., Dubuc, A. M., Conlin, L., Hickey, S. E., Lamb, A. N., ... Guidelines, C. (2018). Yield of additional genetic testing after chromosomal microarray for diagnosis of neurodevelopmental disability and congenital anomalies: A clinical practice resource of the American college of medical genetics and genomics (ACMG). Genetics in Medicine, 20(10), 1105-1113https://doi. org/10.1038/s41436-018-0040-6

Zhou, J., \& Parada, L. F. (2012). PTEN signaling in autism spectrum disorders. Current Opinion in Neurobiology, 22(5), 873-879. https://doi.org/10.1016/j.conb.2012.05.004

Publisher's Note Springer Nature remains neutral with regard to jurisdictional claims in published maps and institutional affiliations. 